\title{
Publisher's Note: High intensity neutrino oscillation facilities in Europe [Phys. Rev. Accel. Beams 16, 021002 (2013)]
}

T. R. Edgecock, O. Caretta, T. Davenne, C. Densam, M. Fitton, D. Kelliher, P. Loveridge, S. Machida, C. Prior, C. Rogers, M. Rooney, J. Thomason, D. Wilcox, E. Wildner, I. Efthymiopoulos, R. Garoby, S. Gilardoni, C. Hansen, E. Benedetto, E. Jensen, A. Kosmicki, M. Martini, J. Osborne, G. Prior, T. Stora, T. Melo Mendonca, V. Vlachoudis, C. Waaijer, P. Cupial, A. Chance, A. Longhin, J. Payet, M. Zito, E. Baussan, C. Bobeth, E. Bouquerel, M. Dracos, G. Gaudiot, B. Lepers, F. Osswald, P. Poussot, N. Vassilopoulos, J. Wurtz, V. Zeter, J. Bielski, M. Kozien, L. Lacny, B. Skoczen, B. Szybinski, A. Ustrzycka, A. Wroblewski, M. Marie-Jeanne, P. Balint, C. Fourel, J. Giraud, J. Jacob, T. Lamy, L. Latrasse, P. Sortais, T. Thuillier, S. Mitrofanov, M. Loiselet, Th. Keutgen, Th. Delbar, F. Debray, C. Trophine, S. Veys, C. Daversin, V. Zorin, I. Izotov, V. Skalyga, G. Burt, A. C. Dexter, V. L. Kravchuk, T. Marchi, M. Cinausero, F. Gramegna, G. De Angelis, G. Prete, G. Collazuol, M. Laveder, M. Mazzocco, M. Mezzetto, C. Signorini, E. Vardaci, A. Di Nitto, A. Brondi, G. La Rana, P. Migliozzi, R. Moro, V. Palladino, N. Gelli, D. Berkovits, M. Hass, T. Y. Hirsh, M. Schaumann, A. Stahl, J. Wehner, A. Bross, J. Kopp, D. Neuffer, R. Wands, R. Bayes, A. Laing, P. Soler, S. K. Agarwalla, A. Cervera Villanueva, A. Donini, T. Ghosh, J. J. Gomez Cadenas, P. Hernandez, J. Martin-Albo, O. Mena, J. Burguet-Castell, L. Agostino, M. Buizza-Avanzini, M. Marafini, T. Patzak, A. Tonazzo, D. Duchesneau, L. Mosca, M. Bogomilov, Y. Karadzhov, R. Matev, R. Tsenov, E. Akhmedov, M. Blennow, M. Lindner, T. Schwetz,

E. Fernandez Martinez, M. Maltoni, J. Menendez, C. Giunti, M. C. Gonzalez Garcia, J. Salvado, P. Coloma, P. Huber, T. Li, J. Lopez Pavon, C. Orme, S. Pascoli, D. Meloni, J. Tang, W. Winter, T. Ohlsson, H. Zhang, L. Scotto-Lavina, F. Terranova, M. Bonesini, L. Tortora, A. Alekou, M. Aslaninejad, C. Bontoiu, A. Kurup, L. J. Jenner, K. Long, J. Pasternak, J. Pozimski, J. J. Back, P. Harrison, K. Beard, A. Bogacz, J. S. Berg, D. Stratakis, H. Witte, P. Snopok, N. Bliss, M. Cordwell, A. Moss, S. Pattalwar, and M. Apollonio (Received 21 June 2016; published 8 July 2016)

DOI: 10.1103/PhysRevAccelBeams.19.079901

This paper was published online on 20 February 2013 with a misspelling in the author list. The 50th author's name should read as "A. Ustrzycka." The name has been corrected online as of 28 June 2016.

Published by the American Physical Society under the terms of the Creative Commons Attribution 3.0 License. Further distribution of this work must maintain attribution to the author(s) and the published articles title, journal citation, and DOI. 